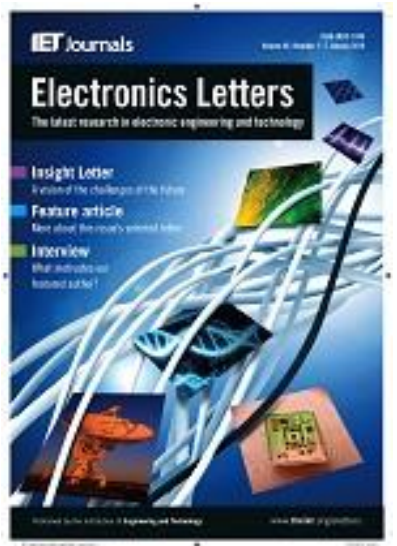

\title{
Iterative receiver based on the EGC for massive MIMO schemes using SC-FDE modulations
}

\begin{abstract}
:
This Letter considers the use of massive multiple-input, multiple-output (MIMO) combined with single-carrier with frequency-domain equalisation (SC-FDE) modulations. A low-complexity iterative frequency-domain receiver based on the equal gain combining (EGC) approach is proposed. This receiver does not require matrix inversions and has excellent performance, which can be very close to the matched filter bound after just a few iterations, even when the number of receive antennas is not very high.
\end{abstract}

Keywords: multiple-input, multiple-output (MIMO); single-carrier with frequencydomain equalisation (SC-FDE); equal gain combining (EGC) 